\title{
Safety of dental extractions in patients on dual antiplatelet therapy - a meta-analysis
}

\author{
Michał Zabojszcz ${ }^{1}$, Krzysztof P. Malinowski², Agnieszka Janion-Sadowska ${ }^{1}$, Theodoros Lillis ${ }^{3}$, \\ Antonios Ziakas ${ }^{4}$, Agnieszka Sławska ${ }^{5}$, Marianna Janion $^{1}$, Zbigniew Siudak $^{1}$ \\ ${ }^{1}$ Faculty of Medicine and Health Science, Jan Kochanowski University, Kielce, Poland \\ ${ }^{2}$ Institute of Public Health, Faculty of Health Sciences, Jagiellonian University Medical College, Krakow, Poland \\ ${ }^{3}$ Department of Dentoalveolar Surgery, Implant surgery and Radiology, Faculty of Dentistry, School of Health Sciences, Aristotle University \\ of Thessaloniki, Greece \\ ${ }^{4}$ Department of Cardiology, Aristotle University of Thessaloniki, AHEPA University Hospital, Thessaloniki, Greece \\ ${ }^{5}$ Department of Interventional Cardiology, GVM Carint, Ostrowiec Świętokrzyski, Poland
}

Adv Interv Cardiol 2019; 15, 1 (55): 68-73

DOI: https://doi.org/10.5114/aic.2019.83773

\begin{abstract}
A bstract
Introduction: Safety of dental extractions in patients on chronic antiplatelet therapy either with only acetylsalicylic acid (ASA) or clopidogrel or with both combined has been a matter of debate, with no clearly conclusive studies published.

Aim: To perform a meta-analysis of published observational studies in order to study the effect of single and double antiplatelet therapy in comparison to controls on the occurrence of immediate local bleeding complications during dental extractions.

Material and methods: PubMed/Scopus/Embase database search revealed 22 papers (13 original and 9 review), 3 of which were finally included in the meta-analysis. Phrases searched: dual[All Fields] AND antiplatelet[All Fields] AND ("therapy"[Subheading] OR "therapy"[All Fields] OR "therapeutics"[MeSH Terms] OR "therapeutics"[All Fields]) AND ("tooth extraction"[MeSH Terms] OR (“tooth"[All Fields] AND "extraction"[All Fields]) OR “tooth extraction"[All Fields]).

Results: The overall event incidence (bleeding complication after extraction) in the entire population was $1.59 \%$ (42 events in 2637 patients). As compared to the control group, the use of double antiplatelet therapy DAPT was associated with on odd ratio OR of 40.23 (95\% Cl: 4.37-370.36) increase in risk of bleeding events occurrence $(p=0.0011)$. Significant heterogeneity was observed $\left(p<0.001 ; r^{2}\right.$ of $\left.76.7 \%\right)$.

Conclusions: Dental extractions following strict procedural protocols in patients on double antiplatelet therapy with clopidogrel and ASA are associated with an additional risk of immediate local bleeding complications.
\end{abstract}

Key words: tooth extraction, cardiovascular diseases, platelet aggregation.

Su m m a ry

This is the first meta-analysis based on strict enrollment and outcome definition criteria in order to select only homogeneous studies and investigating immediate bleeding complications after dental extractions in patients on single (SAPT) or double (DAPT) antiplatelet therapy. Although the majority of studies report no excess bleeding on DAPT, we have proven that when pooling these data there is an increased risk of immediate bleeding, especially on acetylsalicylic acid and clopidogrel therapy.

\section{Introduction}

The management of dental extractions in patients on single (SAPT) or double antiplatelet therapy (DAPT) by dentists has raised major concerns since these therapies have become more widely used among patients after percutaneous coronary interventions. The number of patients receiving antiplatelet therapies is increasing annually [1]. The duration of these therapies for individual pa-

\section{Corresponding author:}

Zbigniew Siudak MD, PhD, Faculty of Medicine and Health Science, Jan Kochanowski University, Kielce, Poland, phone: +48 883992288 ,

e-mail: zbigniew.siudak@gmail.com

Received: 22.11.2018, accepted: 16.12.2018. 
tients varies but is usually between 1 and 12 months for DAPT and sometimes lifelong for SAPT [2]. Moreover, new generations of more potent antiplatelet drugs have been introduced onto the market and recommended by the European Society of Cardiology (ESC) guidelines [2, 3]. The fear of potential bleeding risk during tooth extraction has been weighed against the fear of acute stent thrombosis, which is strongly linked to discontinuation of DAPT and may become fatal [4].

Safety of dental extractions in patients on chronic antiplatelet therapy either with only acetylsalicylic acid (ASA) or clopidogrel or with both combined has been a matter of debate for some time [5]. No clearly conclusive studies have been published either way, mainly due to low sample size. However, the results of the majority of them have proven the safety of dental extractions in the setting of antiplatelet drugs in comparison to naïve patients [6]. Unfortunately, only observational studies are available since no randomized clinical trials have been conducted.

In 2016 a position paper of cardiologists and dentists was published, which encourages maintaining single or double antiplatelet therapy in patients undergoing small surgical dental procedures [7]. The consensus advocates that management in such clinical scenarios should follow the scarce evidence that has been provided so far rather than putting patients at risk of cardiovascular events.

\section{Aim}

The aim of this paper was to perform a meta-analysis of published observational studies that met specific inclusion/exclusion criteria as described in the PICO Scheme below in order to study the effect of single and double antiplatelet therapy in comparison to controls on the occurrence of immediate local bleeding complications during dental extractions.

\section{Material and methods}

A systematic review and meta-analysis was conducted in order to answer the following focused question: "Do patients on antiplatelet therapy (single or dual) who undergo dental extractions without discontinuation of treatment when compared with healthy subjects experience more post-extraction bleeding complications?"

The following PICO scheme was applied.

\section{Population}

Inclusion criteria: Patients with dual antiplatelet therapy (ASA and clopidogrel) or single antiplatelet therapy (ASA or clopidogrel) during tooth/teeth extraction who did not terminate or withheld their treatment for the extraction procedure.

Exclusion criteria: Liver disease, alcoholism, current anticoagulant therapy, current non-steroidal anti-inflammatory drugs (NSAID) therapy or any chronic disease that could impact bleeding after extraction. Patients on ticlopidine (not recommended by the ESC guidelines) and prasugrel (only 2 patients in the entire meta-analysis population) and ticagrelor (no patients) were excluded from the analysis.

\section{Intervention}

Simple tooth/teeth extractions (without raising a mucoperiosteal flap) with the use of local anesthesia which treated primarily with local hemostatic measures that are usually applied by dentists (bite on a pressure gauze pad and/or placement of local hemostatic agent such as oxidative cellulose gauze, collagen or gelatin sponge, etc.)

\section{Controls}

Patients with no antiplatelet therapy at the time of dental extraction.

\section{Outcome}

Postoperative bleeding event occurrence as defined by Lockhart et al. or based on this definition [8]. Prolonged immediate bleeding was defined as the need to use hemostatic gauze when blood extended beyond the tooth socket after 30 min of biting on a pressure pack. Studies where only VAS (Visual Assessment Scale) for bleeding severity was used were not included.

On May $3^{\text {rd }} 2018$ a PubMed, Scopus and EMBASE database search with no time-of-publication limits was performed. The search was conducted independently by two investigators. In case of any discrepancies the committee was organized with a third member to minimize selection bias. We used the following English phrases:

- dual[All Fields] AND antiplatelet[All Fields] AND ("therapy"[Subheading] OR "therapy"[All Fields] OR "therapeutics"[MeSH Terms] OR "therapeutics"[All Fields]) AND ("tooth extraction"[MeSH Terms] OR ("tooth"[All Fields] AND "extraction"[All Fields]) OR "tooth extraction"[All Fields]) and

- dual[All Fields] AND antiplatelet[All Fields] AND ("therapy"[Subheading] OR "therapy"[All Fields] OR "therapeutics"[MeSH Terms] OR "therapeutics"[All Fields]) AND ("tooth extraction"[MeSH Terms] OR ("tooth"[All Fields] AND "extraction"[All Fields]) OR "tooth extraction"[All Fields] OR ("dental”[All Fields] AND “extraction"[All Fields]) OR “dental extraction"[All Fields]). We also searched the database with the additional words "aspirin", "clopidogrel", "prasugrel" and "ticagrelor" in the search phrase instead of "dual antiplatelet therapy" so as to extract all possible publications.

Due to the established strict PICO definition, for inclusion in the analysis, only papers which fulfilled patient inclusion and exclusion criteria as well as outcome measures described below were finally considered suitable to be used in qualitative and quantitative analysis. Only studies with all intergroup comparisons (DAPT, SAPT with 

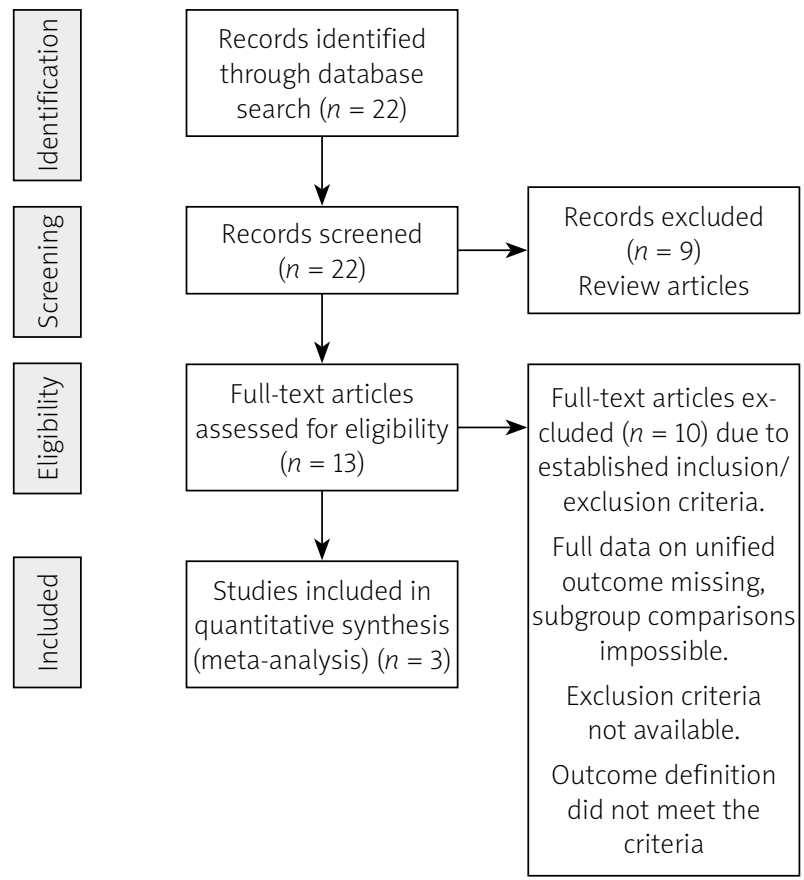

Figure 1. PRISMA 2009 Flow Diagram

ASA, SAPT with clopidogrel, controls) were included in the analysis. The above-mentioned process is also presented in the PRISMA flowchart (Figure 1). The risk of individual study bias was minimized due to strict study selection criteria and similarity of populations and outcome measurements in studies. Patients selection in all studies was based on consecutive patient (prospective or retrospective) enrollment. No external funding was used to perform this meta-analysis. All three studies entered into meta-analysis were approved by local bioethics committees. External funding was only reported for the Lu et al. study, which was supported in part by a CMRP research grant from the Chang Gung Memorial Hospital, Kaohsiung, Taiwan (CMRPG8C0642) [9].

\section{Statistical analysis}

For each arm of all 3 trials the number of patients who experienced bleeding was collected as well as the total number of patients. The summary for all patients with bleeding events was presented as a percentage. The standard errors from arm-based data were transformed into standard errors for pairwise comparisons and the percentages were recalculated into odds ratios with the control as a reference group. Each of the considered trials presented results for each of the considered arms (SAPT with ASA, SAPT with clopidogrel, DAPT and control); hence all comparisons were direct head-to-head comparisons. The similarity of selected trials was assured with the detailed PICO scheme that allowed application of rigorous criteria to study population, design, outcome measures as well as patients' characteristics and treatment - well known effect modifiers. Heterogeneity was assessed using Cochrane's Q; the percentage of variation across studies due to heterogeneity rather than chance was expressed using the $R^{2}$ statistic. Random or fixed arm-based models were calculated depending on observed heterogeneity. Results were presented both with and without continuity correction (default value of $0.5)$ as sensitivity analysis. Results were visualized using forest and network plots [10-13]. Statistical analysis was performed by experienced statistician (KM) in $R$ 3.3.2 ( $R$ Core Team (2017). R: A language and environment for statistical computing. R Foundation for Statistical Computing, Vienna, Austria.) using the packages "meta" 4.92 "metafor" 2.0-0 and "netmeta" 0.9-8 as well as Review Manager [14]. $P$-values less than 0.05 were considered to indicate statistical significance.

\section{Results}

The search revealed 22 papers (21 in English and 1 in German). Nine papers were excluded from further analysis because they were review articles. Altogether 13 full papers were evaluated by the authors for potential meta-analysis. In total, three studies were finally included in this meta-analysis (Tables I, II). The three studies that were analyzed included 2673 patients and observed 42 patients with immediate bleeding events (1.59\%). All these events were minor bleeding complications. Numerical data used for this meta-analysis were extracted and retrieved from the final PDF versions of all 3 papers (tables and text) and were available for all evaluated subgroups. Each of the analyzed studies included all considered arms; hence head-to-head comparisons were possible. Without applying continuity correction the analysis showed that patients receiving SAPT with clopidogrel and DAPT with ASA and clopidogrel had significantly greater odds for bleeding than the control patients. Such a result was not observed in terms of SAPT with ASA (Figure 2). Applying the continuity correction the result holds for and DAPT with ASA and clopidogrel only. The odds for SAPT with ASA as well as SAPT with clopidogrel were not significant when compared to the control group (Figure 3). The overall heterogeneity was significant with $p$-value for Cochrane's $Q$ test of 0.0002 . As compared to the control group, the use of DAPT was associated with on OR of 40.23 (95\% Cl: 4.37-370.36) increase in risk of bleeding events occurrence $(p=0.0011)$. Significant heterogeneity was observed ( $p<0.001 ; l^{2}$ of $76.7 \%$ ).

\section{Discussion}

The key finding of this meta-analysis is that there seems to be a significant increase in local bleeding complications in patients undergoing dental extractions treated with double antiplatelet therapy with ASA and clopidogrel in comparison to controls, which is contradictory to previously published results, which however were each time based on a small sample [9, 15-18]. 
Table I. Papers included in the meta-analysis

\begin{tabular}{|c|c|c|c|c|c|}
\hline Author & Study type & IRB approval & Funding & Groups & Outcome \\
\hline Lillis et al. & $\begin{array}{l}\text { Prospective } \\
\text { observational }\end{array}$ & Yes & None reported & $\begin{array}{l}\text { SAPT with ASA, SAPT with } \\
\text { clopidogrel, DAPT, Controls }\end{array}$ & $\begin{array}{c}\text { Lockhart definition of bleed } \\
\text { ing applied [11] }\end{array}$ \\
\hline Lu et al. & $\begin{array}{l}\text { Retrospective } \\
\text { registry }\end{array}$ & Yes & $\begin{array}{c}\text { The study was supported } \\
\text { in part by a CMRP research } \\
\text { grant from the Chang } \\
\text { Gung Memorial Hospital, } \\
\text { Kaohsiung, Taiwan (CMRP- } \\
\text { G8C0642) }\end{array}$ & $\begin{array}{l}\text { SAPT with ASA, SAPT with } \\
\text { clopidogrel, DAPT, Controls }\end{array}$ & $\begin{array}{l}\text { Immediate bleeding defini- } \\
\text { tion based on Lockhart [11] }\end{array}$ \\
\hline Bajkin et al.* & $\begin{array}{l}\text { Prospective } \\
\text { observational }\end{array}$ & Yes & None reported & $\begin{array}{l}\text { SAPT with ASA, SAPT with } \\
\text { clopidogrel, DAPT, Controls }\end{array}$ & $\begin{array}{c}\text { Lockhart definition of bleed } \\
\text { ing applied [11] }\end{array}$ \\
\hline
\end{tabular}

${ }^{*}$ Patients with ticlopidine (not recommended by guidelines anymore) and prasugrel (only 2 cases) were not included in the analysis. SAPT - single antiplatelet therapy, ASA - acetylsalicylic acid, DAPT - dual antiplatelet therapy.

Table II. Results of selected trials

\begin{tabular}{|c|c|c|c|c|c|}
\hline Study & Group & $\begin{array}{c}\text { Number of patients } \\
\text { with bleeding }\end{array}$ & $\begin{array}{l}\text { Total number } \\
\text { of patients }\end{array}$ & $\begin{array}{c}\text { Percentage of patients } \\
\text { with events (\%) }\end{array}$ & OR $(95 \% \mathrm{Cl})$ vs. control* \\
\hline \multirow[t]{4}{*}{ Lu et al. } & SAPT ASA & 2 & 185 & 1.1 & $1.60(0.3474-7.3491)$ \\
\hline & SAPT clopidogrel & 2 & 65 & 3.1 & $4.64(0.9960-21.6277)$ \\
\hline & DAPT & 1 & 24 & 4.2 & $6.36(0.7811-51.7263)$ \\
\hline & Control & 10 & 1472 & 0.7 & - \\
\hline \multirow[t]{4}{*}{ Lillis et al. } & SAPT ASA & 1 & 42 & 2.4 & $6.46(0.5739-72.7878)$ \\
\hline & SAPT clopidogrel & 1 & 36 & 2.8 & $7.57(0.6701-85.5487)$ \\
\hline & DAPT & 22 & 33 & 66.7 & $530(110.7251-2536.9137)$ \\
\hline & Control & 2 & 532 & 0.4 & - \\
\hline \multirow[t]{4}{*}{ Bajkin et al. } & SAPT ASA & 0 & 84 & 0.0 & $1.25(0.0245-63.5811)^{\wedge}$ \\
\hline & SAPT clopidogrel & 0 & 20 & 0.0 & $5.15(0.0993-266.8422)^{\wedge}$ \\
\hline & DAPT & 1 & 39 & 2.6 & $8.22(0.3279-206.1241)$ \\
\hline & Control & 0 & 105 & 0.0 & - \\
\hline
\end{tabular}

${ }^{*}$ Adjusted for pairwise comparisons; ${ }^{\wedge}$ with continuity correction.

There is a limited amount of published data concerning the topic of our analysis. The studies which are available are only observational prospective and retrospective samples of patients. On the other hand, even though our sample comprised over 2000 cases, the overall observed incidence rate of local immediate bleeding complications was very low (1.6\%). A recently published meta-analysis concluded similarly that DAPT vs placebo carries additional risk for postoperative bleeding [19]. The main difference with our analysis is the inclusion of studies with various outcome measures defining bleeding. We also used only studies with direct SAPT, DAPT and control group comparisons.

Even though the majority of available observational data seem to support the safety of dental extractions in antiplatelet regimes, many dentists still fear the effect the antiplatelet therapy may have on bleeding complications [20, 21]. It is vital for the community of cardiologists to provide evidence-based recommendations for dentists (e.g. review or meta-analysis of existing data) in order to diminish the practice of possible discontinuation of DAPT or SAPT for dental extractions [7]. On the other hand, with the introduction of new, more potent antiplatelet drugs such as prasugrel and ticagrelor there is only one direct comparison in dental extractions, which revealed that prasugrel was associated with a considerably longer bleeding time than clopidogrel [22]. A recent study, which was not included in the meta-analysis due to conflicting outcome definitions, concluded that using ticagrelor as part of DAPT does not increase the bleeding risk after extraction [23].

In our opinion based on the published data and the results of this meta-analysis, the crucial factor influencing potential bleeding complications is not the antiplatelet therapy itself (no platelet testing for its real efficacy in the discussed papers was evaluated) but the extraction procedure and the management of the wound $[5,7,8]$. The operators' skills and following strict dental 


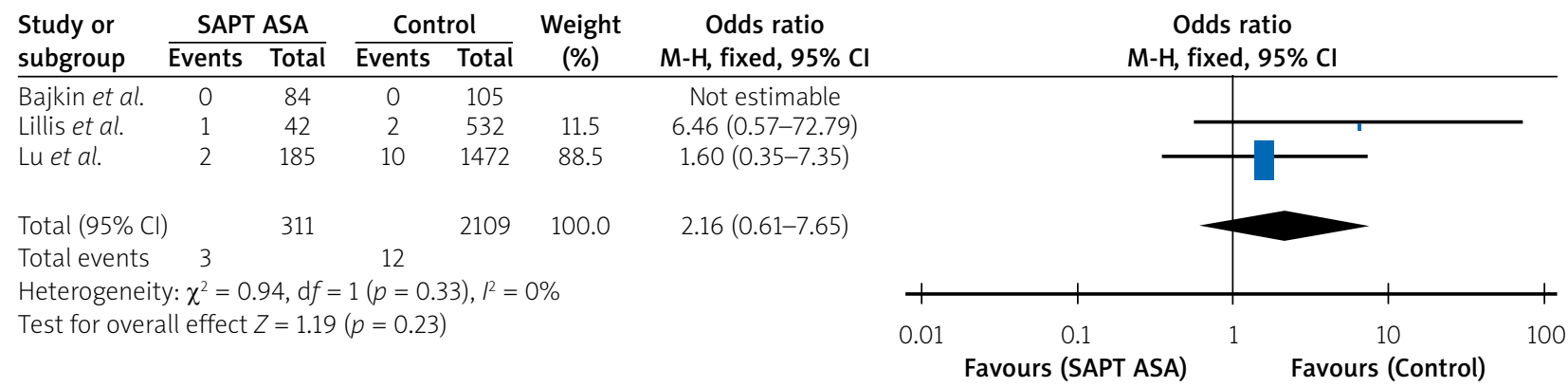

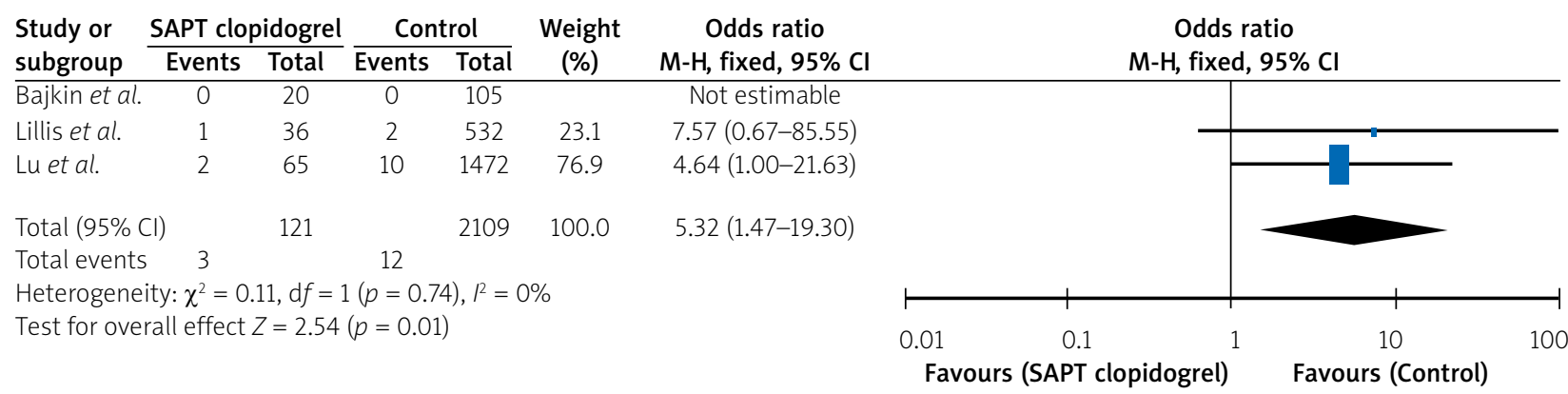

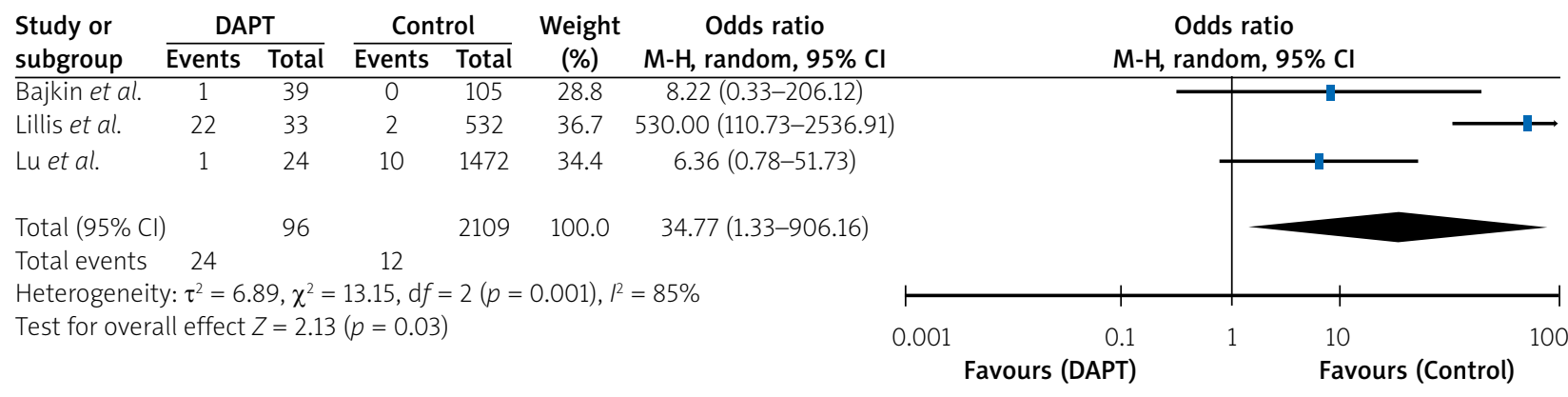

Figure 2. Intergroup comparisons performed in the meta-analysis

Antiplatelet therapy

Odds ratio $(95 \% \mathrm{Cl})$

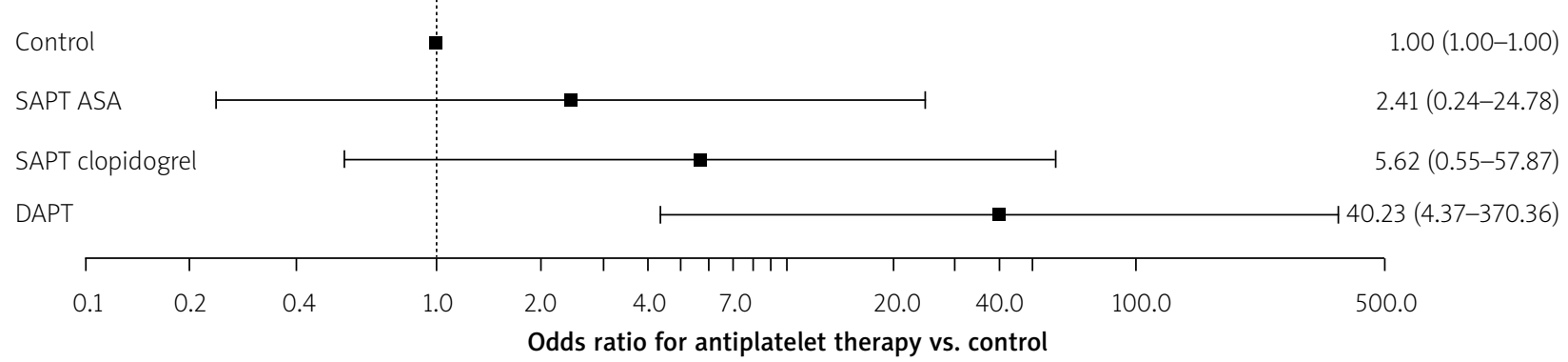

Figure 3. Meta-analysis results presented as OR and $95 \% \mathrm{Cl}$ in controls vs. SAPT or DAPT

algorithms to minimize the risk of bleeding (gauze pads, thrombin, sutures etc.) irrespective of the concomitant antiplatelet therapy play a key role in the success of dental extractions and preventing bleeding complications. It is also worth noting that the observed bleeding events were minor and did not carry additional risk for patients' safety in all 3 papers included in the meta-analysis.

In order to minimize publication and data bias, we included in the meta-analysis only papers with a homo- geneous outcome and clear extraction procedure descriptions and definitions. Only papers with all inter-group comparisons available with control groups were included in the meta-analysis. Statistical measures were undertaken so as to minimize the heterogeneity of the data. However, the small number of trials made it impossible to construct informative funnel plots and the test for symmetry was not performed following the recommendation by Sterne et al. [24]. 
The main limitation of this analysis is the lack of randomized clinical trials which could be included in the meta-analysis. However, in the face of a lack of randomized data we felt it is reasonable to investigate the existing data. It is also troublesome to pool published data since different outcome measures are applied in various papers. It also seems that the baseline profile of patients may play a potential role in the incidence of bleeding, e.g. the frequency of renal failure [18]. We tried to minimize this bias and finally included 3 papers so as to follow strict inclusion/exclusion criteria and similar outcome measures used in each manuscript.

\section{Conclusions}

Dental extractions following strict procedural protocols in patients on double antiplatelet therapy with clopidogrel and ASA are associated with an additional risk of immediate local bleeding complications. Based on our results there seems to be no controversy over the safety of dental extraction on SAPT. However, new studies with prasugrel and ticagrelor are desired in order to confirm the effect also in the new generation of antiplatelet agents, preferably in a randomized manner.

\section{Conflict of interest}

The authors declare no conflict of interest.

\section{References}

1. Legutko J, Siudak Z, Parma R, et al. Poland: coronary and structural heart interventions from 2010 to 2015. Eurolntervention 2017; 13: Z51-4.

2. Windecker S, Kolh P, Alfonso F, et al. 2014 ESC/EACTS Guidelines on myocardial revascularization: The Task Force on Myocardial Revascularization of the European Society of Cardiology (ESC) and the European Association for Cardio-Thoracic Surgery (EACTS) Developed with the special contribution of the European Association of Percutaneous Cardiovascular Interventions (EAPCI). Eur Heart J 2014; 35: 2541-619.

3. Tantry US, Navarese EP, Myat A, Gurbel PA. Selection of p2y12 inhibitor in percutaneous coronary intervention and/or acute coronary syndrome. Prog Cardiovasc Dis 2018; 60: 460-70.

4. Russo JJ, Goodman SG, Bagai A, et al. Duration of dual antiplatelet therapy and associated outcomes following percutaneous coronary intervention for acute myocardial infarction: contemporary practice insights from the Canadian Observational Antiplatelet Study. Eur Heart I Qual Care Clin Outcomes 2017; 3: 303-11.

5. Schächinger V. Care of patients after coronary stent implantation: what is important in practice? Internist (Berl) 2007; 48: 1365-74.

6. Schreuder WH, Peacock ZS. Antiplatelet therapy and exodontia. J Am Dent Assoc 2015; 146: 851-6.

7. Pruszczyk P, Ciurzyński M, Opolski G, et al. Dental cardio common position for dealing anticoagulation in patients undergoing dental procedures. Kardiol Pol 2016; 74: 87-98.

8. Lockhart PB, Gibson J, Pond SH, Leitch J. Dental management considerations for the patient with an acquired coagulopathy.
Part 2: Coagulopathies from drugs. Br Dent J 2003; 195: 495501.

9. Lu SY, Tsai CY, Lin LH, Lu SN. Dental extraction without stopping single or dual antiplatelet therapy: results of a retrospective cohort study. Int J Oral Maxillofac Surg 2016; 45: 1293-8.

10. Jackson D, White IR, Riley RD. Quantifying the impact of between-study heterogeneity in multivariate meta-analyses. Stat Med 2012; 31: 3805-20.

11. van Houwelingen, HC, Arends, LR, Stijnen, T. Advanced methods in meta-analysis: multivariate approach and meta-regression. Stat Med 2002; 21: 589-624.

12. Schwarzer G, Carpenter JR, Rücker G. Meta-Analysis with R (Use-R!). Springer International Publishing 2015, Switzerland.

13. Napeñas JJ, Hong CH, Brennan MT, et al. The frequency of bleeding complications after invasive dental treatment in patients receiving single and dual antiplatelet therapy. J Am Dent Assoc 2009; 140: 690-5.

14. RevMan; Version 5.3. Copenhagen: The Nordic Cochrane Centre, The Cochrane Collaboration, 2014.

15. Bajkin BV, Urosevic IM, Stankov KM, et al. Dental extractions and risk of bleeding in patients taking single and dual antiplatelet treatment. Br J Oral Maxillofac Surg 2015; 53: 39-43.

16. Lillis T, Ziakas A, Koskinas K, et al. Safety of dental extractions during uninterrupted single or dual antiplatelet treatment. Am J Cardiol 2011; 108: 964-7.

17. Buhatem Medeiros F, Pepe Medeiros de Rezende N, Bertoldi Franco J, et al. Quantification of bleeding during dental extraction in patients on dual antiplatelet therapy. Int J Oral Maxillofac Surg 2017; 46: 1151-7.

18. Yanamoto S, Hasegawa T, Rokutanda S, et al. Multicenter retrospective study of the risk factors of hemorrhage after tooth extraction in patients receiving antiplatelet therapy. J Oral Maxillofac Surg 2017; 75: 1338-43.

19. Li L, Zhang W, Yang Y, et al. Dental management of patient with dual antiplatelet therapy: a meta-analysis. Clin Oral Investig 2018 Aug 25. doi: 10.1007/s00784-018-2591-y.

20. Rai R, Mohan B, Babbar V, Dang N. Practices and perceptions of doctors for patients on anti-platelets during dental surgery: a national survey. J Maxillofac Oral Surg 2014; 13: 249-52.

21. van Diermen DE, Bruers JJ, Hoogstraten J, et al. Treating dental patients who use oral antithrombotic medication: a survey of dentists in the Netherlands. J Am Dent Assoc 2011; 142: 137682.

22. Dézsi BB, Koritsánszky L, Braunitzer G, et al. Prasugrel versus clopidogrel: a comparative examination of local bleeding after dental extraction in patients receiving dual antiplatelet therapy. J Oral Maxillofac Surg. 2015; 73: 1894-900.

23. Doganay O, Atalay B, Karadag E, et al. Bleeding frequency of patients taking ticagrelor, aspirin, clopidogrel, and dual antiplatelet therapy after tooth extraction and minor oral surgery. J Am Dent Assoc 2018; 149: 132-8.

24. Sterne JAC, Sutton AJ, loannidis JP, et al. Recommendations for examining and interpreting funnel plot asymmetry in meta-analyses of randomised controlled trials. BMJ 2011; 343: d4002. 